\title{
Platelet-rich fibrin in oral surgical procedures: a systematic review and meta-analysis
}

João Vitor dos Santos Canellas, Paulo Jose D'Albuquerque Medeiros,

Carlos Marcelo da Silva Figueredo, Ricardo Guimaraes Fischer, Fabio Gamboa Ritto

Rio de Janeiro State University

\section{Corresponding author}

João Vitor dos Santos Canellas

Rio de Janeiro State University,

Rua Boulevard 28 de Setembro,

157 Vila Isabel,

Rio de Janeiro, RJ 20551-030,

Brazil.

drcanellas@icloud.com

5521971507053

Short Title: Platelet-rich fibrin in oral surgery 


\begin{abstract}
The effect of platelet-rich fibrin (PRF) for enhancing the healing after oral surgical interventions is still a matter of debate. The purpose of this study was to identify what indications has PRF been shown effective in oral surgical procedures. A comprehensive literature search was performed up to 2017 on PubMed/MEDLINE, Cochrane Library, Web of Science, Scopus and LILACS databases and grey literature. The full-text of potentially relevant studies were reviewed and only randomized clinical trials (RCT) were included. A total of 559 studies were found, of which 30 were included for qualitative analysis and 13 for quantitative analysis. Three review authors assessed the risk of bias independently. The available literature suggests that PRF performs positive effect to improve alveolar preservation on extraction sockets and around dental implants. The qualitative analysis showed a significantly better effect of PRF to promote bone regeneration for alveolar cleft reconstruction. The meta-analysis for third molar surgery showed a decrease in prevalence of alveolar osteitis. PRF increased implant stability 1 week and 1 month after surgery $(P=.003$ and .005$)$. Due to the lack of studies with low risk of bias and a limited number of patients available, further RCTs are needed to confirm these results.
\end{abstract}

Keywords: platelet-rich fibrin; oral surgical procedures; oral surgery; bone healing. 


\section{Introduction}

Since the first description by Choukroun ${ }^{1}$ in 2000 , platelet-rich fibrin (PRF) has become an important surgical adjuvant in oral surgical procedures. In fact, only after the publication of five important articles ${ }^{2-6}$ in 2006 , this biomaterial gained highlight in the field of dental research. To date, more than 500 scientific articles have been published on this subject. Some of the possible recommendations to use this biomaterial in oral surgical procedures are third molar surgery, alveolar ridge preservation after tooth extractions, sinus lift procedure, repair of alveolar cleft, dental implants, surgical treatment of medication-related osteonecrosis of the jaw and treatment of oroantral communications. ${ }^{7-13}$

PRF is a second generation of platelet concentrate ${ }^{14}$ produced without biochemical blood manipulation, and basically constituted for three key parameters: first, the presence of platelets and its activated growth factors that are substantial embedding into the fibrin matrix during the natural polymerization process ${ }^{5}$; second, the presence of leucocytes and its cytokines that contribute for anti-infectious action and for immune regulation of the healing process ${ }^{6,}$ 15; third, the density and complex organization of the fibrin matrix architecture produced by a natural polymerization, without addition of any anticoagulant and gelling agent. ${ }^{16}$

The strong fibrin architecture distinguishes it from other kinds of platelets concentrates, like platelet-rich plasma (PRP). This fibrin matrix seems responsible for the slow release of growth factors during proliferation stage of wound healing, over a period of 7-14 days, and it is composed of thin fibers with micropores that can serve 
as a scaffold for cell migration and differentiation. PRF is an important reservoir of numerous growth factors to promote angiogenesis, such as transforming growth factor $\beta$ (TGF- $\beta$ ) and vascular endothelial growth factor (VEGF). There are still large amounts of platelet-derived growth factors (PDGFs) in platelet $\alpha$-granules, which act as an essential regulator for collagen production and mesenchymal cell migration and proliferation. ${ }^{5,16-18}$

Although many indications to the use PRF in oral surgical procedures have been suggested, due to its biological potential to improve wound healing process, it is necessary to observe which of these oral surgical indications have been proven effective under an evidence-based analysis, leading to its recommended use to enhance tissue/bone formation. The objective of this systematic review was to answer the following focused question: what indications has platelet-rich fibrin been shown effective for oral surgical procedures? 


\section{Materials and Methods}

The protocol for this systematic review was registered on PROSPERO (CRD42017078422). ${ }^{19}$ The PRISMA statement (Preferred Reporting Items for Systematic Review and Meta-Analyses) was used to ensure higher methodological quality of the article.20 The PICOS strategy was used for the research question construction: $(\mathrm{P})$ patients or population: health patients requiring oral surgical procedures (orthognathic surgery, mandibular third molar surgery, surgical treatment for medication-related osteonecrosis of the jaw, sinus lift procedure, treatment of oroantral communications, alveolar ridge preservation after tooth extractions, alveolar cleft reconstruction and dental implants); (I) intervention: use of autologous PRF combined or not with other biomaterial; (C) comparator or control group: none treatment or another biomaterial combination without PRF; $(\mathrm{O})$ Orthognathic surgery (pain, swelling, bone healing or postoperative complications), third molar surgery (pain, swelling, bone healing or alveolar osteitis), osteonecrosis of the jaw (prevalence of complete resolution), sinus lift procedure (bone regeneration, soft tissue healing or success rate of dental implants), oroantral communications (success rate, pain, swelling or time of surgery). alveolar ridge preservation (sockets dimensions, bone density, histomorphometric analysis or resorption of marginal bone), alveolar cleft grafting (bone volume, bone density or bone dimensions), dental implants (marginal bone change around dental implant or implant stability); (S) Study design: studies in humans, including only RCT. There were no language restrictions. An electronic search of the MEDLINE/PubMed database, Cochrane Library, Web of Science database, Scopus database and Latin American and Caribbean Health Sciences Literature database (LILACS) was conducted. The search strategy used both medical subject headings terms $(\mathrm{MeSH})$ and free-text 
words. The search detailed PICOS framework are shown in supplementary material file to allow adequate reproduction of this study.

No language or time restrictions were applied for screening process. The search was limited to human studies and the last electronic search was performed on the September $16^{\text {th }} 2017$. The following trial registry platforms were screened to find studies from the 'grey literature': ClinicalTrials.gov (http://www.clinicaltrials.gov) and EU Clinical Trials Register (https://www.clinicaltrialsregister.eu). The reference lists of the articles identified were cross-checked. A manual search was performed in the following relevant journals of oral and maxillofacial surgery: International Journal of Oral and Maxillofacial Surgery, Journal of Oral and Maxillofacial Surgery, Journal of Cranio-Maxillofacial Surgery, Oral and Maxillofacial Surgery Clinics of North America, Oral Surgery Oral Medicine Oral Pathology Oral Radiology and British Journal of Oral \& Maxillofacial Surgery. Subsequently, the articles were imported into Endnote $\mathrm{X} 7$ software (Thompson Reuters, Philadelphia, PA, USA) where duplicates were removed.

Studies that used anticoagulants, gelling agents or any other biochemical blood handling to prepare the platelet concentrate were excluded. Additionally, controlled clinical trials, retrospective studies, duplicate study, editorial letters, historical reviews, in vitro studies, cohort and observational studies were excluded. Finally, the articles were excluded when the full text was not found or in case of unpublished work that the authors could not be contact to give more informations. 
Titles and abstracts of the screening studies were independently evaluated by two reviewers (JVC and FGR). The full-text articles were obtained for those studies that the authors' judgment was considered relevant or uncertain regarding selection criterions. The level of agreement between the review authors was calculated by Kappa statistic. Value of Kappa between 0.40 and 0.59 is considered fair agreement, between 0.60 and 0.74 is a good agreement and 0.75 or more is considered excellent agreement. Disagreement regarding inclusion was resolved by discussion with third author (PJM). The Primary outcome for orthognathic surgery was bone healing; for mandibular third molar surgery was alveolar osteitis $(A O)$; for surgical treatment of osteonecrosis of the jaw was prevalence of complete resolution; for sinus lift procedure was the percentage of new bone formation; for oroantral communications was the success rate; for alveolar ridge preservation was sockets dimensions; for alveolar cleft grafting was the percentage of newly formed bone and for dental implants was the marginal bone resorption. The secondary outcomes for orthognathic surgery were pain, swelling and postoperative complications; for mandibular third molar were pain, swelling and bone healing; for sinus lift procedure were the success rate of dental implants installed and soft tissue healing; for oroantral communications were pain, swelling and time of surgery; for alveolar ridge preservation were bone density and marginal bone levels; for alveolar cleft grafting were bone density, bone volume or bone dimensions and for dental implants was implant stability.

The Cochrane Collaboration tool was used for assessing the risk of bias in included studies. Three review authors (JVC, FGR, PJM) assessed independently the risk of 
bias. The reviewers were blinded to informations about the article, such as the journal, the authors, the institution and direction of the results and the magnitude.

Any disagreements between the authors were resolved by discussion. In the risk of bias analysis, sequence generation was considered adequate if the method used to generate the allocation sequence was described in sufficient details to allow an assessment of whether it should produce comparable groups. Allocation concealment was considered adequate if the method used to conceal the allocation sequence was sufficient detailed to determine whether intervention allocations could not have been foreseen in advance of, or during enrollment. The blinding of participants and outcome assessors were evaluated as well as the incomplete outcome, selective outcome reporting and other sources of bias. The assessment of the risk of bias was undertaken as follows: low risk of bias when all of the domains judged to be at low risk of bias; unclear risk of bias, when one or more domains judged to be at unclear risk of bias; and high risk of bias when one or more domains judged to be at high risk of bias.

Qualitative and quantitative synthesis were performed and the meta-analysis was accomplished when the outcome variables were sufficiently homogenous. In the absence of statistical heterogeneity, Chi-square test $\left(\chi^{2}\right)>0.10$ and I-squared $\left(I^{2}\right)$ $<50 \%$, a fixed-effects model was used. The random-effect model was used to incorporate heterogeneity among studies and provide a more conservator pooled intervention effect when statistical heterogeneity was present. Review Manager version 5.3 software (The Nordic Cochrane Centre, The Cochrane Collaboration,

Commented [JVC2]:

Review 3

Reviewers' comment: Although derived by Higgins the Isquared value is not commonly referred to as the

I'Higgins Index'. It would be preferable to call this simply I-squared. 
Copenhagen, Denmark, 2014) was used for the meta-analysis computations. For the variables with considerable methodological and statistical heterogeneity, a metaanalysis could not be conducted, thus only a descriptive synthesis was performed. 


\section{Results}

The PRISMA flow diagram of the screening and selection process is presented in Figure 1. A total of 1073 articles were identified initially and after the exclusion of duplicates, 559 records remained. After the eligibility process 512 records were excluded and 47 full-text articles were obtained. After full-text reading of these 47 articles, 30 randomized control trials (RCTs) fulfilled the inclusion criteria and were selected for qualitative analysis, 14 articles about third molar surgery ${ }^{8}, 21-33,5$ articles about sinus lift procedure ${ }^{34-38}, 5$ articles $^{12,} 39-42$ and 1 conference abstract ${ }^{43}$ about alveolar preservation, 3 articles about dental implants ${ }^{44-46}$ and 2 articles about treatment of cleft patients. ${ }^{11,47}$ For quantitative analysis 13 articles were included, 9 articles about third molar $8,25,26,28,29,32,48-50,2$ articles about sinus $\operatorname{lift}^{35}, 38$ and 2 articles about dental implants ${ }^{46,51}$. Seventeen articles were excluded and the reasons for exclusion were as follows: 4 non-randomized controlled clinical trial ${ }^{52-55} ; 4$ studies which outcomes of interest were not reported $56-59 ; 2$ studies that used other PRF protocol $^{60,61}$; 1 study which did not test PRF62; 1 retrospective study ${ }^{63} ; 2$ prospective studies $^{64,65 ;} 1$ study that used the same patients sample as other studies ${ }^{66} ; 1$ full text was not found ${ }^{67} ;$ and 1 study was written in persa and not translated ${ }^{68}$. The value of Kappa was 0.84 , thus it was considered an excellent agreement. The characteristics of the included articles are shown in Table 1 and 2.

None of the trials included in this review were assessed as at low risk of bias for all the domains. Sixteen trials were assessed as unclear risk of bias. The remaining 14 trials were assessed as high overall risk of bias because each of these trials was at high risk of bias in one or more domains. (Figure 2) 


\section{Mandibular Third Molar Surgery}

In the primary outcome (AO) 3 studies were analyzed (Table 3). ${ }^{8,29,50}$ There were 226 extractions (113 test, 113 control) in 172 patients. No relevant clinical heterogeneity was observed among the studies, the values of Chi-square test $\left(\chi^{2}\right)$ and $\mid$-squared $\left(I^{2}\right)$ were 0.84 and $0 \%$ respectively. The quantitative analysis showed a decrease in prevalence of $\mathrm{AO}$ (odds ratio $0.33,95 \%$ confidence interval $0.14,0.76$; $Z=2.61, P=0.009)$ presented in Figure 3 . Three secondary outcomes were analyzed, pain (Table 4), swelling (Table 5) and bone healing (Table 6). The level of pain after surgery included 9 articles $22,24-26,28-30,32,50$ of which 6 showed a favorable effect of PRF $(p<0.05)$ to reduce postoperative pain $24,26,28-30,50$. A meta-analysis was applied for first, second, third days and for the sum of the first seven days. A favorable effect of PRF in the 3rd day (Figure 4) and in the sum of values in the first week (Figure 5) was observed, and no difference between groups in 1st (Figure 6)and 2nd day (Figure 7) was found. The values of Chi-square test $\left(\chi^{2}\right)$ and Isquared $\left(1^{2}\right)$ demonstrated a high heterogeneity among the studies for the first, second and third days analysis, and a low heterogeneity for the sum of the first seven days in the analysis. To evaluate postoperative swelling, 6 articles were included ${ }^{24-26,}$ $28,29,32$ of which 3 showed a favorable effect of PRF $(p<0.05)$ to reduce edema ${ }^{24,25,29}$ (Table 5). The meta-analysis to evaluate swelling after 1, 2 and 3 days (Figures 8, 9 and 10 respectively) showed low heterogeneity among the studies, and only beneficial overall effect of PRF on facial swelling after 3 days was noted (Figure 10).

\section{Commented [JVC3]:}

Review 3

Reviewers' comment: Although derived by Higgins the Isquared value is not commonly referred to as the I'Higgins Index'. It would be preferable to call this simply I-squared.

\section{Commented [JVC4]:}

Review 3

Reviewers' comment: The reported confidence intervals in the text use a '-' symbol to separate out the upper and lower limits. Given that some limits are negative, it is slightly confusing as to when this symbol is indicating a negative number and when it is implying the gap

between the two figures. To avoid confusion, an alternative symbol (e.g. ',') could be used instead.

Commented [JVC5]:
Review 3
Reviewers' comment: Although derived by Higgins the I-
squared value is not commonly referred to as the
I'Higgins Index'. It would be preferable to call this simply
I-squared.


Lastly, about effect of PRF over bone healing, 7 studies were included in the qualitative analyze $24,27,29,30,33,48,49,5$ studies $^{24,27, ~ 29, ~ 30, ~ 33 ~ e v a l u a t e d ~ t h e ~ b o n e ~ h e a l i n g ~}$ through radiographs of which 4 studies $^{27,}$ 29, 30, 33 showed that PRF was significantly better in promoting bone regeneration after third molar extraction $(p<0.05)($ Table 7$)$. Due to the high methodological heterogeneity, it was not possible to perform a metaanalysis for these studies. Two studies ${ }^{48,} 49$ evaluated bone healing through scintigraphy, and for these studies a meta-analysis did not find difference in osteoblastic activity when PRF was used or not (mean difference $-0.03,95 \%$ confidence interval $-0.53,0.46 ; Z=0.13, P=0.90)$ Figure 11.

\section{Sinus Lift Procedure}

In the primary outcome (percentage of new bone formation) 4 studies were analyzed (Table 2). ${ }^{34,35,38,69}$ Two studies ${ }^{35,} 38$ showed no difference to use PRF mixture with bone substitute or bone substitute alone, one study ${ }^{69}$ showed similar effect of PRF membrane and conventional collagen membrane (Bio-Gide), when they were used as a membrane at the lateral osteotomy window, and one study ${ }^{34}$ showed that PRF reduced the healing time, favoring optimal bone cicatrization (Table 3 ). In the secondary outcome, one study evaluated the soft tissue and showed the favorable effects of PRF on wound healing, however this diference was not statistically significant (Table 7). The values of Chi-square test $\left(\chi^{2}\right)$ and I-squared $\left(I^{2}\right)$ demonstrated a moderate heterogeneity among the studies, $\mathrm{P}=0.20$ and $\mathrm{I}^{2}=40 \%$. The quantitative analysis showed that there was no significant difference in the mean percentage of new bone formations when PRF was added or not to bone substitute

\section{Commented [JVC6]:}

Review 3

Reviewers' comment: The reported confidence intervals in the text use a '-' symbol to separate out the upper

and lower limits. Given that some limits are negative, it is slightly confusing as to when this symbol is indicating a negative number and when it is implying the gap

between the two figures. To avoid confusion, an alternative symbol (e.g. ',') could be used instead.

Commented [JVC7]:
Review 3
Reviewers' comment: Although derived by Higgins the I-
squared value is not commonly referred to as the
I'Higgins Index'. It would be preferable to call this simply
I-squared.


(standard mean difference $0.29,95 \%$ confidence interval $-0.73,1.30 ; Z=0.55, P=$ 0.58) (Figure 12).

\section{Alveolar Ridge Preservation}

Three studies showed a positive effect after comparing PRF (alone or combined with graft material) to control group (no filling material or graft without PRF), and found that PRF improved the preservation of the alveolus and resulted in less bone resorption. ${ }^{12,}$ 39, 42 One study compared PRF with beta-tri-calcium phosphate and showed the similar effect in alveolar ridge preservation for both biomaterials. ${ }^{41}$ Only one study ${ }^{70}$ did not find statistical significance between PRF group and control group, although the PRF group demonstrated faster bone healing compared with the control in this study. The authors also concluded that PRF accelerated soft-tissue healing on the first 4 weeks. ${ }^{70}$ The primary outcomes are presented in Table 3 and secondary outcomes in Table 8. Due to the high methodological heterogeneity, it was not possible to perform a meta-analysis for these studies. One conference abstract ${ }^{43}$ concluded that bone density was not statistically significant different after comparing PRF group and control, however PRF group demonstrated superiority in maintaining bone width. The authors were contacted to answer detailed informations about the unpublished work, unfortunately we did not receive the data in time to include it in this systematic review.

\section{Dental Implants and Cleft Patients}

\section{Commented [JVC8]:}

Review 3

Reviewers' comment: The reported confidence intervals

in the text use a '-' symbol to separate out the upper

and lower limits. Given that some limits are negative, it

is slightly confusing as to when this symbol is indicating

a negative number and when it is implying the gap

between the two figures. To avoid confusion, an

alternative symbol (e.g. ',') could be used instead. 
Only one $\mathrm{RCT}^{11}$ evaluating the use of PRF in treatment of cleft patients was found. In this study, the mean percentage of newly formed bone, after unilateral maxillary alveolar cleft reconstruction, was significantly higher in patients that received PRF combined with autogenous bone graft than in those that received autogenous bone graft alone (Table 3). The mean bone density of the newly formed bone was similar in both groups $(p>0.05)$.

Two articles evaluated the effect of PRF on peri-implant marginal bone resorption. ${ }^{44}$, 46 These studies confirmed the beneficial effect of PRF in reducing the peri-implant bone resorption following dental extraction with immediate implant placement, and following implant placement in partially edentulous patients. Due to the high methodological heterogeneity, it was not possible to perform a meta-analysis for these studies. (Table 3) The secondary outcome for dental implants evaluation, the effect of PRF on implant stability quotient (ISQ), measured by resonance frequency analysis, was evaluated for two articles. ${ }^{45}, 46$ Both studies found that the use of PRF increases implant stability during initial healing period. The overall effect of PRF on ISQ after 1 week and 1 month are presented in the Figures 13 and 14 respectively.

This systematic review did not find RCTs evaluating the use of PRF for orthognathic surgery, osteonecrosis surgical treatment and for closure of oroantral communications. 


\section{Discussion}

Certainly, PRF has gained tremendous highlight in recents years due to its capacity to successfully regenerate either soft or hard tissues, enhancing new blood vessels (angiogenesis), and tissue formation during the healing. Some advantages of PRF over PRP are the lack of anti-coagulants blood, which results in a strong fibrin matrix, and considerable growth factor that may be released over 10 to 14 days period. The theory is that the combination of host cells, strong fibrin matrix, and growth factors, acts to produce a faster wound healing. The objective of this systematic review was to evaluate what indications has platelet-rich fibrin been shown effective for oral surgical procedures.

A total of 8 types of surgical interventions routinely performed in oral and maxillofacial surgery were analyzed in this systematic review. Currently, the use of PRF in mandibular third molar surgery has been broadly studied, and 3 recents systematic reviews ${ }^{71-73}$ showed a beneficial effect of PRF on preventing dry sockets within the first 7 days. However, the study of He et al. ${ }^{73}$ included 1 article ${ }^{49}$ that presented 2 cases of infection after surgery ( 3 weeks and 2 months), that should not have been included in the meta-analysis to evaluate AO. Furthermore the authors mixed RCT and retrospective studies to estimate the effect size, thus decreasing the accuracy of the analysis. The present meta-analysis included only RCT, and found that PRF can be used to reduce the incidence of $A O$ in the first week after mandibular third molar surgery, diminishing the risk of $A O$ by $62 \%$ when compared with no treatment. 
Others outcomes evaluated in mandibular third molar surgery were pain, swelling and bone healing. The pain evaluation should be analyzed with caution because this meta-analysis showed an important heterogeneity among the included studies. Some of the possible reasons that we should considered before interpreting the results are: sex-based differences in pain perception, type of analgesic therapy utilized in each study, ethnic disparities in progression of pain-related conditions, carry-across effect in pain analysis when the authors used a split-month design, and difficulty to measure pain due to its multifaceted and subjective nature. The results of this metaanalysis showed a positive effect of PRF on postoperative pain confirming the results of other recents systematic reviews. ${ }^{72,73}$

Our results supported that PRF was more effective to reduce swelling in the third day although only 3 studies have been included and it demands more studies to confirm the real effect. In the bone healing evaluation, the qualitative analysis observed a favorable effect of PRF on hard tissue cicatrization, similarly to other systematic reviews that evaluated platelets concentrates. ${ }^{74-76}$ Only one systematic review showed no beneficial role of PRF in bone healing after extraction of mandibular third molars, however this study presented some limitations in the methodology and need to be cautiously interpreted.77, 78 The methodological differences among studies made it impossible to perform a meta-analysis to bone density, although the qualitative analysis showed a beneficial effect of PRF. The meta-analysis about the effect of PRF on osteoblastic activity was performed and no difference between PRF and control group was observed. Osteoblastic activity failed to assess the real effect 
of PRF in the bone-healing because it is not sensitive to bone mass evaluation. This is a surrogate endpoint which can lead to misinterpretation. ${ }^{78}$

Currently, PRF has been used in sinus lift procedure either in combination with other biomaterial or as a sole filling material.64, 79 The release of blood-derived growth factors facilitates angiogenesis and increases blood flow into the sinus cavity favoring and accelerating bone healing. This systematic review did not find any RCT evaluating PRF as sole filling material. The qualitative analysis showed 3 studies that compared the use of xenograft materials with or without $\mathrm{PRF}^{34,35,38}$, and no statistic difference was detected. Nonetheless, 2 of these studies ${ }^{35,38}$ demonstrated a higher percentage of newly formed bone when PRF was used in combination with xenograft material in sinus augmentation. This was probably due to the limited quantity of patients evaluated in the meta-analysis, only 14 patients. Tatullo et al. ${ }^{34}$ showed, through histological analysis of 72 sinus lifts procedures, that PRF reduces the healing time favoring faster bone cicatrization. According to the authors, it is possible to obtain good stability of endosseous implants installed 106 days after sinus augmentation.

PRF has also been utilized to cover the lateral maxillary window during sinus augmentation. One $\mathrm{RCT}^{80}$ investigating 12 sinuses from six patients compare PRF membrane with conventional collagen membrane (Bio-Gide®). The authors concluded that similar amount of bone formation could be achieved after 5 months, when both type of membranes were utilized to cover the lateral window. The main advantages to use PRF in this situation are the low cost, the easy of obtainment, and the totally biocompatibility of the membrane. 
PRF has been suggested to treat Schneiderian membrane perforations during sinus elevation. ${ }^{81}$ PRF is recommended due to it is resorption period of 10 to 14 days, totally biocompatibility, and adequate size to cover most common small perforations. None RCT evaluating PRF for this treatment is available for ethic reasons, since it would be necessary to either create intentional tears on the membrane, or to have an enormous sample to analyze the few cases that would suffer unintentional tears.

The use of PRF has been most frequently studied for alveolar ridge preservations after tooth extractions. Nowadays, we know that none single therapy can completely prevent the alveolar changes after dental extractions, and some amount of bone loss is always expected.82, 83 Pre-clinical studies showed the negative effect of tooth extraction in alveolar dimensions, probably as a result of drastic initial loss in blood supply derived from periodontal ligaments. ${ }^{84}, 85$ In this context, the pro-angiogenic cytokines and growth factors of PRF may improve alveolar bone preservation.

In this systematic review most studies confirmed that PRF decreased the alveolar resorption after dental extractions when used alone or combined with other biomaterials. This result is similar to other recent systematic reviews, ${ }^{75,76,86}$ although these findings should be cautiously interpreted due to the limited amount of the available evidence. Two $\mathrm{RCT}^{12,39}$ evaluating the ridge preservation properties of PRF showed a positive effect in alveolar dimensions preservation. The quantity of bone resorption was similar to the expected for other biomaterials, around $1 \mathrm{~mm}$ bone loss horizontally and vertically. ${ }^{82}$ Hauser et al. showed that the positive effect of PRF 
disappeared when a more invasive surgery was performed, thus, the extraction with mucosal flap elevation should be avoided. Suttapreyasri et al. ${ }^{70}$ did not find statistical difference between PRF and control group in alveolar ridge preservation. Possible factors for this result are some limitations of their study, such as two-dimensional image analysis (radiography), absence of histological analysis, measurement of the resorption of marginal bone at the extraction site without a reliability assessment method for measurement.

One recent conference abstract ${ }^{43}$ confirmed the effect of PRF on bone width preservation, however it was not possible to assess the risk of bias of this study. We contact the corresponding author for clarification and additional informations to assess the risk of bias, however she not responde in time to include the data in this analysis.

Only two $\mathrm{RCT} \mathrm{T}^{11}, 47$ assessing the use of PRF in alveolar cleft reconstruction were found. The first study ${ }^{11}$ analyzed 24 patients with unilateral alveolar cleft submitted to bone reconstruction. Two groups of 12 subjects were analyzed, the first received autologous bone graft plus PRF and the second received only autologous bone graft. PRF significantly increased the percentage of newly formed bone, but no statistical difference was observed when bone density was analyzed. Nonetheless, in spite of the statistical result, bone density was higher in the PRF group. The authors believed that the fibrin matrix could aid the increase of bone formation after the surgery. 
The second study ${ }^{87}$ assessed 2 treatment methods in alveolar cleft reconstruction. The combination of chin symphysis bone, allogeneic bone material and PRF showed similar results compared with the iliac bone graft. In this study the real contribution of PRF in bone healing cannot be measured. In theory, PRF works as a source of growth factors, increasing the vascularization and osteoblastic differentiation. More studies evaluating this effect are necessary to confirm these results.

A frequent topic in discussion regarding PRF is its potential to accelerate osteointegration in dental implant surgery. Although some studies have shown a beneficial effect of PRF, an evidence-based validity to determine the real effect is necessary. The assessment of marginal bone level is one of the most important indicators of implant health. Two studies ${ }^{44,81}$ included in the qualitative analysis confirmed the positive effect of PRF on peri-implant marginal bone resorption. Some limitations of these studies are radiographic analysis and short follow-up period.

The present meta-analysis showed increased ISQ values during the early healing (1st week and 1 month) when PRF was utilized inside the osteotomy. However, it is necessary to cautiously interpret these results. Only 2 studies, with limited sample size and performed by the same group were included in this meta-analysis. Furthermore, the subgroup analysis of Oncu et al. ${ }^{51}$ found statistically significant differences between PRF and control group only in type 2 bone, and there were no difference between groups with other types of bone. The question whether PRF can augment the value of ISQ during osteointegration period remains unanswered, and more RCT with adequate methodological design are necessary. 
Some limitations of this systematic review was that the majority of studies showed insufficient informations to permit judgment of "yes" or "no" about the sequence generation process and allocation concealment. Therefore, the unclear status could introduce selection bias, and in this case, systematic differences between baseline characteristics of the compared groups may be present. Another problem of this systematic review was that all included studies showed no trial registration, the only exception was the study of Gülşen et. al. ${ }^{32}$ One of the concerns about no registrations is that results with statistically non-significant might have been selectively withheld from the final publication, thus introducing a reporting bias. The results should be interpreted with caution because a total of 14 studies (out of 30 ) showed a high risk of bias, therefore the plot of intervention effect estimates may be imprecise. Finally, the method used to estimate bone density and new bone formation were too diverse to allow good comparison. To analyze theses variants, more studies using histomorphometry and tomography instead of radiography are desired.

A total of twelve meta-analysis were performed in this study and the majority of these were a mixture of split-month design and parallel design. A more appropriate analysis of cross-over trials should take the individual correlation into account using a paired analysis ${ }^{88}$, measuring the difference for each participant between the intervention and control, and taking the mean of these within-person differences. However the limited amount of informations in the primary studies made this approach impossible and the precision of results might be a little compromised. A unit-of-analysis error can arise from this approach leading to overestimation or underestimation of the each 
study's weight. Future RCTs with split-mouth design are encouraged to provide more information about paired analysis and correlation coefficient, thus permitting a more precise meta-analysis.

In summary, the available literature found suggest that PRF may perform better wound healing when it is used in oral surgical procedures. The most consistent indications found in this systematic review are: 1) to preserve alveolar dimensions after tooth extractions; and 2) to reduce dry sockets incidence after mandibular third molar surgery. PRF is very a promising biomaterial to enhanced alveolar cleft reconstruction and to reduce peri-implant marginal bone resorption, although a limit quantity of information about these indications are available. The effect of PRF in sinus augmentation showed no advantage in percentage of final new bone formation, however the healing time might be reduced when PRF is added. Due to the lack of studies with low risk of bias and homogeneous methodology to permit a more precise meta-analysis, further RCTs are needed to confirm the evidence for these surgical indications.

\section{Declarations}

Funding: None

Competing Interests: None declared

Ethical Approval: Not required

Patient Consent: Not required 


\section{References}

1. Choukroun J, Adda F, Schoeffler C, Vervelle A. Une opportunité en paroimplantologie: le PRF. Implantodontie. 2000;42:55-62.

2. Choukroun J, Diss A, Simonpieri A, Girard MO, Schoeffler C, Dohan SL, et al. Platelet-rich fibrin (PRF): A second-generation platelet concentrate. Part V: Histologic evaluations of PRF effects on bone allograft maturation in sinus lift. Oral Surgery Oral Medicine Oral Pathology Oral Radiology and Endodontics. 2006;101(3):299-303.

3. Choukroun J, Diss A, Simonpieri A, Girard MO, Schoeffler C, Dohan SL, et al. Platelet-rich fibrin (PRF): a second-generation platelet concentrate. Part IV: clinical effects on tissue healing. Oral surgery, oral medicine, oral pathology, oral radiology, and endodontics. 2006;101(3):e56-60.

4. Dohan DM, Choukroun J, Diss A, Dohan SL, Dohan AJ, Mouhyi J, et al. Platelet-rich fibrin (PRF): a second-generation platelet concentrate. Part I: technological concepts and evolution. Oral surgery, oral medicine, oral pathology, oral radiology, and endodontics. 2006;101(3):e37-44.

5. Dohan DM, Choukroun J, Diss A, Dohan SL, Dohan AJ, Mouhyi J, et al. Platelet-rich fibrin (PRF): a second-generation platelet concentrate. Part II: plateletrelated biologic features. Oral surgery, oral medicine, oral pathology, oral radiology, and endodontics. 2006;101(3):e45-50.

6. Dohan DM, Choukroun J, Diss A, Dohan SL, Dohan AJ, Mouhyi J, et al. Platelet-rich fibrin (PRF): a second-generation platelet concentrate. Part III: leucocyte activation: a new feature for platelet concentrates? Oral surgery, oral medicine, oral pathology, oral radiology, and endodontics. 2006;101(3):e51-5. 
7. Mazor Z, Horowitz RA, Del Corso M, Prasad HS, Rohrer MD, Ehrenfest DMD. Sinus floor augmentation with simultaneous implant placement using Choukroun's platelet-rich fibrin as the sole grafting material: A radiologic and histologic study at 6 months. Journal of periodontology. 2009;80(12):2056-64.

8. Eshghpour M, Dastmalchi $\mathrm{P}$, Nekooei $\mathrm{AH}$, Nejat $\mathrm{A}$. Effect of platelet-rich fibrin on frequency of alveolar osteitis following mandibular third molar surgery: A doubleblinded randomized clinical trial. Journal of Oral and Maxillofacial Surgery. 2014;72(8):1463-7.

9. Kim JW, Kim SJ, Kim MR. Leucocyte-rich and platelet-rich fibrin for the treatment of bisphosphonate-related osteonecrosis of the jaw: a prospective feasibility study. The British journal of oral \& maxillofacial surgery. 2014;52(9):854-9. 10. Boora P, Rathee M, Bhoria M. Effect of Platelet Rich Fibrin (PRF) on Periimplant Soft Tissue and Crestal Bone in One-Stage Implant Placement: A Randomized Controlled Trial. J Clin Diagn Res. 2015;9(4):Zc18-21.

11. Shawky H, Seifeldin SA. Does Platelet-Rich Fibrin Enhance Bone Quality and Quantity of Alveolar Cleft Reconstruction? The Cleft palate-craniofacial journal : official publication of the American Cleft Palate-Craniofacial Association. 2016;53(5):597-606.

12. Temmerman A, Vandessel J, Castro A, Jacobs R, Teughels W, Pinto N, et al. The use of leucocyte and platelet-rich fibrin in socket management and ridge preservation: a split-mouth, randomized, controlled clinical trial. Journal of clinical periodontology. 2016;43(11):990-9.

13. Bilginaylar K. The Use of Platelet-Rich Fibrin for Immediate Closure of Acute Oroantral Communications: An Alternative Approach. Journal of oral and 
maxillofacial surgery : official journal of the American Association of Oral and Maxillofacial Surgeons. 2017.

14. Dohan Ehrenfest DM, Rasmusson L, Albrektsson T. Classification of platelet concentrates: from pure platelet-rich plasma (P-PRP) to leucocyte- and platelet-rich fibrin (L-PRF). Trends Biotechnol. 2009;27(3):158-67.

15. Pirraco RP, Reis RL, Marques AP. Effect of monocytes/macrophages on the early osteogenic differentiation of hBMSCs. J Tissue Eng Regen Med. 2013;7(5):392-400.

16. Dohan Ehrenfest DM, Del Corso M, Diss A, Mouhyi J, Charrier JB. Threedimensional architecture and cell composition of a Choukroun's platelet-rich fibrin clot and membrane. Journal of periodontology. 2010;81(4):546-55.

17. Kobayashi E, Fluckiger L, Fujioka-Kobayashi M, Sawada K, Sculean A, Schaller B, et al. Comparative release of growth factors from PRP, PRF, and advanced-PRF. Clinical oral investigations. 2016;20(9):2353-60.

18. Fujioka-Kobayashi M, Miron RJ, Hernandez M, Kandalam U, Zhang $\mathrm{Y}$, Choukroun J. Optimized Platelet-Rich Fibrin With the Low-Speed Concept: Growth Factor Release, Biocompatibility, and Cellular Response. J Periodontol. $2017 ; 88(1): 112-21$.

19. Canellas JV, Medeiros PJ, Figueredo CM, Fischer R, Ritto F. Has platelet-rich fibrin been shown effective for oral and maxillofacial surgical procedures? A systematic review and meta-analysis. PROSPERO 2017 CRD42017078422 [Available from: http://www.crd.york.ac.uk/PROSPERO/display_record.php?ID=CRD42017078422. 
20. Moher D, Liberati A, Tetzlaff J, Altman DG, Group P. Preferred reporting items for systematic reviews and meta-analyses: the PRISMA statement. BMJ. 2009;339:b2535.

21. Gurbuzer B, Pikdoken L, Tunali M, Urhan M, Kucukodaci Z, Ercan F. Scintigraphic evaluation of osteoblastic activity in extraction sockets treated with platelet-rich fibrin. J Oral Maxillofac Surg. 2010;68(5):980-9.

22. Ruga E, Gallesio C, Boffano P. Platelet-rich fibrin and piezoelectric surgery: A safe technique for the prevention of periodontal complications in third molar surgery. Journal of Craniofacial Surgery. 2011;22(5):1951-5.

23. Baslarli O, Tumer C, Ugur O, Vatankulu B. Evaluation of osteoblastic activity in extraction sockets treated with platelet-rich fibrin. Med Oral Patol Oral Cir Bucal. 2015;20(1):e111-6.

24. Kumar N, Prasad K, Ramanujam L, Ranganath R, Dexith J, Chauhan A. Evaluation of treatment outcome after impacted mandibular third molar surgery with the use of autologous platelet-rich fibrin: A randomized controlled clinical study. Journal of Oral and Maxillofacial Surgery. 2015;73(6):1042-9.

25. Ozgul O, Senses F, Er N, Tekin U, Tuz HH, Alkan A, et al. Efficacy of platelet rich fibrin in the reduction of the pain and swelling after impacted third molar surgery: Randomized multicenter split-mouth clinical trial. Head and Face Medicine. $2015 ; 11(1)$.

26. Uyanik LO, Bilginaylar K, Etikan I. Effects of platelet-rich fibrin and piezosurgery on impacted mandibular third molar surgery outcomes. Head \& face medicine. 2015;11:25. 
27. Yelamali T, Saikrishna D. Role of platelet rich fibrin and platelet rich plasma in wound healing of extracted third molar sockets: a comparative study. Journal of maxillofacial and oral surgery. 2015;14(2):410-6.

28. Bilginaylar K, Uyanik LO. Evaluation of the effects of platelet-rich fibrin and piezosurgery on outcomes after removal of impacted mandibular third molars. $\mathrm{Br} \mathrm{J}$ Oral Maxillofac Surg. 2016;54(6):629-33.

29. Dutta SR, Passi D, Singh P, Sharma S, Singh M, Srivastava D. A randomized comparative prospective study of platelet-rich plasma, platelet-rich fibrin, and hydroxyapatite as a graft material for mandibular third molar extraction socket healing. National journal of maxillofacial surgery. 2016;7(1):45-51.

30. Kumar YR, Mohanty S, Verma M, Kaur RR, Bhatia P, Kumar VR, et al. Platelet-rich fibrin: the benefits. The British journal of oral \& maxillofacial surgery. 2016;54(1):57-61.

31. Al-Hamed FS, Al-Saleh MAQ, Tawfik MA, Abdelfadil E. Efficacy of PlateletRich Fibrin after Mandibular Third Molar Extraction: A Systematic Review and MetaAnalysis Reply. Journal of Oral and Maxillofacial Surgery. 2017;75(8):1577-8.

32. Gulsen U, Senturk MF. Effect of platelet rich fibrin on edema and pain following third molar surgery: a split mouth control study. BMC Oral Health. 2017;17(1):79.

33. Varghese MP, Manuel S, Kumar S. Potential for Osseous Regeneration of Platelet-Rich Fibrin-A Comparative Study in Mandibular Third Molar Impaction Sockets. Journal of Oral and Maxillofacial Surgery. 2017;75(7):1322-9.

34. Tatullo M, Marrelli M, Cassetta M, Pacifici A, Stefanelli LV, Scacco S, et al. Platelet Rich Fibrin (P.R.F.) in reconstructive surgery of atrophied maxillary bones: 
clinical and histological evaluations. International journal of medical sciences. $2012 ; 9(10): 872-80$

35. Zhang Y, Tangl S, Huber CD, Lin Y, Qiu L, Rausch-Fan X. Effects of Choukroun's platelet-rich fibrin on bone regeneration in combination with deproteinized bovine bone mineral in maxillary sinus augmentation: a histological and histomorphometric study. Journal of cranio-maxillo-facial surgery : official publication of the European Association for Cranio-Maxillo-Facial Surgery. 2012;40(4):321-8.

36. Gassling V, Purcz N, Braesen JH, Will M, Gierloff M, Behrens E, et al. Comparison of two different absorbable membranes for the coverage of lateral osteotomy sites in maxillary sinus augmentation: a preliminary study. Journal of cranio-maxillo-facial surgery : official publication of the European Association for Cranio-Maxillo-Facial Surgery [Internet]. 2013; 41(1):[76-82 pp.]. Available from: http://onlinelibrary.wiley.com/o/cochrane/clcentral/articles/842/CN-

00839842/frame.html.

37. Gurler G, DelilbaSi C. Effects of leukocyte-platelet rich fibrin on postoperative complications of direct sinus lifting. Minerva Stomatologica. 2016;65(4):207-12

38. Cömert Kiliç S, Güngörmüş M, Parlak SN. Histologic and histomorphometric assessment of sinus-floor augmentation with beta-tricalcium phosphate alone or in combination with pure-platelet-rich plasma or platelet-rich fibrin: A randomized clinical trial. Clinical implant dentistry and related research. 2017.

39. Hauser F, Gaydarov N, Badoud I, Vazquez L, Bernard JP, Ammann P. Clinical and Histological Evaluation of Postextraction Platelet-rich Fibrin Socket Filling: A Prospective Randomized Controlled Study. Implant Dentistry. 2013;22(3):295-303. 
40. Suttapreyasri S, Leepong N. Influence of Platelet-Rich Fibrin on Alveolar Ridge Preservation. Journal of Craniofacial Surgery. 2013;24(4):1088-94.

41. Das S, Jhingran R, Bains VK, Madan R, Srivastava R, Rizvi I. Socket preservation by beta-tri-calcium phosphate with collagen compared to platelet-rich fibrin: A clinico-radiographic study. European journal of dentistry. 2016;10(2):264-76. 42. Thakkar DJ, Deshpande NC, Dave DH, Narayankar SD. A comparative evaluation of extraction socket preservation with demineralized freeze-dried bone allograft alone and along with platelet-rich fibrin: A clinical and radiographic study. Contemporary clinical dentistry. 2016;7(3):371-6.

43. Sununliganon L, Jiumkim A, Narkbuakaew W. Effect of platelet-rich fibrin in healing socket using cone beam compute tomography analysis. International journal of oral and maxillofacial surgery [Internet]. 2017; Conference: 23rd International Conference on Oral and Maxillofacial Surgery, ICOMS 2017. Hong Kong. 46(pp 352). Available from: http://onlinelibrary.wiley.com/o/cochrane/clcentral/articles/399/CN01381399/frame.html.

44. Boora P, Rathee M, Bhoria M. Effect of Platelet Rich Fibrin (PRF) on periimplant soft tissue and crestal bone in one-stage implant placement: A randomized controlled trial. Journal of Clinical and Diagnostic Research. 2015;9(4):ZC18-ZC21.

45. Öncü E, Alaaddinoğlu EE. The effect of platelet-rich fibrin on implant stability. International Journal of Oral and Maxillofacial Implants. 2015;30(3):578-82.

46. Oncu E, Erbeyoglu AA. Enhancement of Immediate Implant Stability and Recovery Using Platelet-Rich Fibrin. The International journal of periodontics \& restorative dentistry. 2017. 
47. Movahedian Attar B, Naghdi N, Etemadi Sh M, Mehdizadeh M. Chin Symphysis Bone, Allograft, and Platelet-Rich Fibrin: Is the Combination Effective in Repair of Alveolar Cleft? Journal of Oral and Maxillofacial Surgery. 2017;75(5):102635.

48. Gurbuzer B, Pikdoken L, Tunali M, Urban M, Kucukodaci Z, Ercan F. Scintigraphic Evaluation of Osteoblastic Activity in Extraction Sockets Treated With Platelet-Rich Fibrin. Journal of Oral and Maxillofacial Surgery. 2010;68(5):980-9.

49. Baslarli O, Tumer C, Ugur O, Vatankulu B. Evaluation of osteoblastic activity in extraction sockets treated with platelet-rich fibrin. Medicina oral, patologia oral y cirugia bucal. 2015;20(1):e111-e6.

50. Al-Hamed FS, Tawfik MAM, Abdelfadil E. Clinical effects of platelet-rich fibrin (PRF) following surgical extraction of lower third molar. Saudi Journal for Dental Research. 2017;8(1-2):19-25.

51. Oncu E, Alaaddinoglu EE. The effect of platelet-rich fibrin on implant stability. The International journal of oral \& maxillofacial implants. 2015;30(3):578-82.

52. Singh A, Kohli M, Gupta N. Platelet rich fibrin: a novel approach for osseous regeneration. Journal of maxillofacial and oral surgery. 2012;11(4):430-4.

53. Anwandter A, Bohmann S, Nally M, Castro AB, Quirynen M, Pinto N. Dimensional changes of the post extraction alveolar ridge, preserved with Leukocyteand Platelet Rich Fibrin: A clinical pilot study. Journal of dentistry. 2016;52:23-9.

54. Doiphode AM, Hegde P, Mahindra U, Santhosh Kumar SM, Tenglikar PD, Tripathi V. Evaluation of the efficacy of platelet-rich plasma and platelet-rich fibrin in alveolar defects after removal of impacted bilateral mandibular third molars. Journal 
of International Society of Preventive \& Community Dentistry. 2016;6(Suppl 1):S4752.

55. Asaka T, Ohga N, Yamazaki Y, Sato J, Satoh C, Kitagawa Y. Platelet-rich fibrin may reduce the risk of delayed recovery in tooth-extracted patients undergoing oral bisphosphonate therapy: a trial study. Clinical oral investigations. $2017 ; 21(7): 2165-72$.

56. Marenzi G, Riccitiello F, Tia M, di Lauro A, Sammartino G. Influence of Leukocyte- and Platelet-Rich Fibrin (L-PRF) in the Healing of Simple Postextraction Sockets: A Split-Mouth Study. Biomed Research International. 2015.

57. Du Toit J, Siebold A, Dreyer A, Gluckman H. Choukroun Platelet-Rich Fibrin as an Autogenous Graft Biomaterial in Preimplant Surgery: Results of a Preliminary Randomized, Human Histomorphometric, Split-Mouth Study. The International journal of periodontics \& restorative dentistry. 2016;36 Suppl:s75-86.

58. Chakravarthi S. Platelet rich fibrin in the management of established dry socket. Journal of the Korean Association of Oral and Maxillofacial Surgeons. $2017 ; 43(3): 160-5$.

59. Hussain I, Singh S, Jain H, Kumar S, Anand KR, Batool N, et al. A prospective randomised clinical study on evaluation of platelet-rich fibrin versus zinc oxide eugenol in the management of alveolar osteitis. Oral Surgery. 2017.

60. Girish Rao S, Bhat P, Nagesh KS, Rao GH, Mirle B, Kharbhari L, et al. Bone regeneration in extraction sockets with autologous platelet rich fibrin gel. Journal of maxillofacial and oral surgery. 2013;12(1):11-6.

61. Cheng Y, Liu M. Osteogenic effects of concentrated growth factors applied in maxillary sinus floor elevation via a lateral window approach with simultaneous 
implant placement: Study protocol for a single-center randomized controlled trial. Chinese Journal of Tissue Engineering Research. 2017;21(4):574-9.

62. Park JH, Kim JW, Kim SJ. Does the Addition of Bone Morphogenetic Protein 2 to Platelet-Rich Fibrin Improve Healing After Treatment for Medication-Related Osteonecrosis of the Jaw? J Oral Maxillofac Surg. 2017;75(6):1176-84.

63. Hoaglin DR, Lines GK. Prevention of localized osteitis in mandibular thirdmolar sites using platelet-rich fibrin. International journal of dentistry. 2013;2013:875380.

64. Tajima N, Ohba S, Sawase T, Asahina I. Evaluation of sinus floor augmentation with simultaneous implant placement using platelet-rich fibrin as sole grafting material. The International journal of oral \& maxillofacial implants. 2013;28(1):77-83.

65. Norholt SE, Hartlev J. Surgical treatment of osteonecrosis of the jaw with the use of platelet-rich fibrin: a prospective study of 15 patients. International journal of oral and maxillofacial surgery. 2016;45(10):1256-60.

66. Oncu E, Alaaddinoolu E. Effect of platelet-rich fibrin on osseointegration. International journal of oral and maxillofacial surgery [Internet]. 2013; 42(10):[1265-6 pp.]. Available from:

http://onlinelibrary.wiley.com/o/cochrane/clcentral/articles/349/CN-

00875349/frame.html.

67. Kim JH, Lee DW, Ryu DM. Effect of Platelet-Rich Fibrin on Pain and Swelling After Surgical Extraction of Third Molars. Tissue Eng Regen Med. 2011;8(4):80-6. 
68. Amini P, Milani A, Rahmani N. Radiological assessment of using allograft in combination with platelet-rich fibrin in alveolar cleft grafting. Journal of Mazandaran University of Medical Sciences. 2016;26(142):175-85.

69. Gassling V, Purcz N, Braesen JH, Will M, Gierloff M, Behrens E, et al. Comparison of two different absorbable membranes for the coverage of lateral osteotomy sites in maxillary sinus augmentation: A preliminary study. J CranioMaxilloFac Surg. 2013;41(1):76-82.

70. Suttapreyasri S, Leepong N. Influence of platelet-rich fibrin on alveolar ridge preservation. J Craniofac Surg. 2013;24(4):1088-94.

71. Al-Hamed FS, Tawfik MAM, Abdelfadil E, AI-Saleh MAQ. Efficacy of PlateletRich Fibrin After Mandibular Third Molar Extraction: A Systematic Review and MetaAnalysis. Journal of Oral and Maxillofacial Surgery. 2017;75(6):1124-35.

72. Canellas JV, Ritto FG, Medeiros PJD. Evaluation of postoperative complications after mandibular third molar surgery with the use of platelet-rich fibrin: a systematic review and meta-analysis. International journal of oral and maxillofacial surgery. 2017;46(9):1138-46.

73. He Y, Chen J, Huang Y, Pan Q, Nie M. Local Application of Platelet-Rich Fibrin During Lower Third Molar Extraction Improves Treatment Outcomes. Journal of oral and maxillofacial surgery : official journal of the American Association of Oral and Maxillofacial Surgeons. 2017;75(12):2497-506.

74. Del Fabbro M, Bortolin M, Taschieri S. Is autologous platelet concentrate beneficial for post-extraction socket healing? A systematic review. International journal of oral and maxillofacial surgery. 2011;40(9):891-900. 
75. Del Fabbro M, Corbella S, Taschieri S, Francetti L, Weinstein R. Autologous platelet concentrate for post-extraction socket healing: a systematic review. European journal of oral implantology. 2014;7(4):333-44.

76. Del Fabbro M, Bucchi C, Lolato A, Corbella S, Testori T, Taschieri S. Healing of Postextraction Sockets Preserved With Autologous Platelet Concentrates. A Systematic Review and Meta-Analysis. J Oral Maxillofac Surg. 2017;75(8):1601-15.

77. Brignardello-Petersen R. Platelet-rich fibrin might not provide a benefit in healing and complications after third-molar extraction. J Am Dent Assoc. 2017;148(6):e73.

78. Dos Santos Canellas JV, Ritto FG, Medeiros PJD. Efficacy of Platelet-Rich Fibrin After Mandibular Third Molar Extraction: A Systematic Review and MetaAnalysis. Journal of oral and maxillofacial surgery : official journal of the American Association of Oral and Maxillofacial Surgeons. 2017;75(8):1576-7.

79. Mazor Z, Horowitz RA, Del Corso M, Prasad HS, Rohrer MD, Dohan Ehrenfest DM. Sinus floor augmentation with simultaneous implant placement using Choukroun's platelet-rich fibrin as the sole grafting material: a radiologic and histologic study at 6 months. J Periodontol. 2009;80(12):2056-64.

80. Gassling V, Douglas T, Warnke PH, Açil Y, Wiltfang J, Becker ST. Platelet-rich fibrin membranes as scaffolds for periosteal tissue engineering. Clinical oral implants research. 2010;21(5):543-9.

81. Oncu E, Kaymaz E. Assessment of the effectiveness of platelet rich fibrin in the treatment of Schneiderian membrane perforation. Clin Implant Dent Relat Res. 2017;19(6):1009-14. 
82. Morjaria KR, Wilson R, Palmer RM. Bone healing after tooth extraction with or without an intervention: a systematic review of randomized controlled trials. Clinical implant dentistry and related research. 2014;16(1):1-20.

83. locca O, Farcomeni A, Pardinas Lopez S, Talib HS. Alveolar ridge preservation after tooth extraction: a Bayesian Network meta-analysis of grafting materials efficacy on prevention of bone height and width reduction. $J$ Clin Periodontol. 2017;44(1):104-14.

84. Araujo MG, Sukekava F, Wennstrom JL, Lindhe J. Ridge alterations following implant placement in fresh extraction sockets: an experimental study in the dog. Journal of clinical periodontology. 2005;32(6):645-52.

85. Scala A, Lang NP, Schweikert MT, de Oliveira JA, Rangel-Garcia I, Jr., Botticelli D. Sequential healing of open extraction sockets. An experimental study in monkeys. Clin Oral Implants Res. 2014;25(3):288-95.

86. Castro AB, Meschi N, Temmerman A, Pinto N, Lambrechts P, Teughels W, et al. Regenerative potential of leucocyte- and platelet-rich fibrin. Part B: sinus floor elevation, alveolar ridge preservation and implant therapy. A systematic review. Journal of clinical periodontology. 2017;44(2):225-34.

87. Movahedian Attar B, Naghdi N, Etemadi Sh M, Mehdizadeh M. Chin Symphysis Bone, Allograft, and Platelet-Rich Fibrin: Is the Combination Effective in Repair of Alveolar Cleft? Journal of oral and maxillofacial surgery : official journal of the American Association of Oral and Maxillofacial Surgeons. 2017;75(5):1026-35.

88. Elbourne DR, Altman DG, Higgins JP, Curtin F, Worthington HV, Vail A. Metaanalyses involving cross-over trials: methodological issues. Int $\mathrm{J}$ Epidemiol. 2002;31(1):140-9. 


\section{Figure Legends}

Figure 1. PRISMA flow diagram of the screening and selection process.

Figure 2. Risk of bias summary: review authors' judgements.

Figure 3. Forest plot comparing PRF versus control in the prevention of alveolar osteitis.

Figure 4. Forest plot and meta-analysis of pain (3rd day).

Figure 5. Forest plot and meta-analysis of pain (sum of values in the first week).

Figure 6. Forest plot and meta-analysis of pain (1st day).

Figure 7. Forest plot and meta-analysis of pain (2nd day).

Figure 8. Forest plot and meta-analysis of swelling (1st day).

Figure 9. Forest plot and meta-analysis of swelling (2nd day).

Figure 10. Forest plot and meta-analysis of swelling (3rd day).

Figure 11. Forest plot and meta-analysis of osteoblastic activity (1st month).

Figure 12. Forest plot of percentage of new bone formation in sinus lift procedure.

Figure 13. Forest plot of ISQ value after 1 week.

Figure 14. Forest plot of ISQ value after 1 month. 